\title{
Design and first-light performance of TES bolometer arrays for submillimeter spectroscopy with ZEUS-2
}

\author{
Carl Ferkinhoff*a ${ }^{\text {a }}$ Thomas Nikola ${ }^{a}$, Stephen C. Parshley ${ }^{a}$, Gordon J. Stacey ${ }^{a}$, Kent D. Irwin ${ }^{b}$, \\ Hsiao-Mei Cho ${ }^{b}$, Mike Niemack ${ }^{b}$, Mark Halpern ${ }^{c}$, Matthew Hasselfield ${ }^{\mathrm{c}}$, Mandana Amiri ${ }^{\mathrm{c}}$ \\ ${ }^{a}$ Department of Astronomy, Cornell University, Ithaca, NY, USA 14853 \\ ${ }^{\mathrm{b}}$ NIST Boulder, Boulder, CO USA 80305-3337 \\ ${ }^{\mathrm{c}}$ Department of Physics and Astronomy, University of British Columbia, Vancouver, B.C., V6T \\ 1Z1, Canada
}

\begin{abstract}
We have recently commissioned the $2^{\text {nd }}$ generation redshift(z) and Early Universe Spectrometer (ZEUS-2) at the Caltech Submillimeter Observatory. ZEUS-2 is a long-slit grating spectrometer (R 1000) for observations in the submillimeter wavelength regime that is optimized for observations of redshifted far-infrared spectral lines from galaxies in the early universe. Here we report on the design and first light performance of the first TES bolometer array installed in ZEUS-2. This array features 280 pixels each $1.26 \mathrm{~mm}$ square and arranged to provide $\sim 35$ pixel spectra at $\sim 8$ spatial positions on the sky. A 1/4-wavelength back short of 100 micron and gold mesh absorber matching the impedance of free space provides near $90 \%$ quantum efficiency for the 350 and 450 micron telluric windows. Array readout is done using SQUID multiplexers and the Multichannel Electronics. We will also report on the progress to install two additional arrays tuned to provide similar performance across the remaining telluric windows between 200-850 microns.
\end{abstract}

Keywords: ZEUS, CSO, submillimeter, ULIRG, high redshift, far-infrared, star formation, galaxies

\section{INTRODUCTION}

ZEUS-2, is designed to study the star formation history of the Universe from early times until the present epoch continuing the work begun with its predecessor, ZEUS. ${ }^{1}$ ZEUS-2 is optimized for the detection of faint, broad submillimeter and redshifted far-IR (FIR) lines such as the ground state fine-structure lines from $\mathrm{C}^{0}, \mathrm{C}^{+}, \mathrm{O}^{0}$, and the mid$\mathrm{J}$ rotational lines from CO. These lines are important coolants, in some case the dominate coolant, of major phases of the ISM. They are among the brightest lines from a galaxy. By comparing their relative strengths knowledge of both the excitation source and the physical characteristics (i.e. temperature, density) of the gas can be deduced.

\subsection{Previous Work}

Observations with ZEUS on CSO have produced the first extragalactic detection of the ${ }^{13} \mathrm{CO}(6-5)$ line ${ }^{2}$, the first detection of the [OIII] 88 micron line ${ }^{3}$ and [NII] 122 micron line ${ }^{4}$ at redshift greater than 0.05 , and the first detection of the 158 micron line $e^{5}$ of [CII] from between $\mathrm{z}=1-2$. In total ZEUS has made 25 detections of the [CII] 158 micron line in a $\mathrm{z}=1-2$ galaxy survey (12 are described in Stacey et al. $2010^{6}$ ). A mid-J CO survey of local infrared bright (IRB) and ultra-luminous IR galaxies (ULIRG) has also been performed to probe the nature of the activity in nearby galaxies ${ }^{7,8}$

\subsection{Science Goals}

ZEUS-2 will continue and build upon this important and ground breaking work of understanding star formation across cosmic time by working towards three scientific goals (Ferkinhoff et al. 2010, provides a detailed description of the ZEUS-2 science program ${ }^{9}$ ):

\footnotetext{
*carl.ferkinhoff@cornell.edu; phone 1 (607) 255-5891; fax 1 (607) 255-3433; submm.astro.cornell.edu
}

\footnotetext{
Millimeter, Submillimeter, and Far-Infrared Detectors and Instrumentation for Astronomy VI, edited by Wayne S. Holland, Jonas Zmuidzinas, Proc. of SPIE Vol. 8452

845207 @ 2012 SPIE · CCC code: 0277-786X/12/\$18 - doi: 10.1117/12.927237
} 
1. Investigate star formation in the early Universe by detecting redshifted far-IR fine-structure lines from distant ( $\mathrm{z}>0.25-5$, see Figure 2) galaxies. ZEUS-2 is capable of detecting the 158 micron [CII] line from hundreds of SCUBA and Spitzer sources, thousands of sources being identified in Herschel fields, and any high redshift ULIRG detected by the WISE survey. The [CII] line traces the physical extent of a starburst as well as its intensity thereby making the study of the [CII] line in the early universe critical for understanding the star formation history of the Universe.

2. Measure redshifts of optically obscured distant sources using the [CII] 158 micron line. While there is a wealth of sources detectable with ZEUS-2 that already have spectroscopic redshifts, several current missions (e.g. Herschel and WISE) will produce source catalogs containing many infrared bright galaxies with only photometric redshifts. These sources, in addition to many sources from SCUBA and IRAS catalogs lacking spectroscopic redshifts, are prime targets for ZEUS-2 as they are likely enshrouded by dust making optical spectroscopic follow-up difficult if not impossible. ZEUS-2 is uniquely suited for this task due to its large instantaneous bandwidth $(\sim 5 \%$ of a band) and large redshift range accessible across its seven bands.

3. Investigate star formation and molecular gas excitation in nearby galaxies by simultaneously mapping $\mathrm{CO}(7-6),{ }^{13} \mathrm{CO}(6-5),[\mathrm{CI}] 370$ and 609 micron, and [NII] lines to produce maps of the physical conditions of major components of the ISM. These maps will allow us to study how galaxy dynamics (i.e. density waves, resonances, etc.) interact with the ISM and stimulate star formation. Additional high-J CO and ${ }^{13} \mathrm{CO}$ observations will distinguish between cosmic rays, turbulence, and/or X-rays as the dominate source of heating of molecular clouds in ULIRGS.

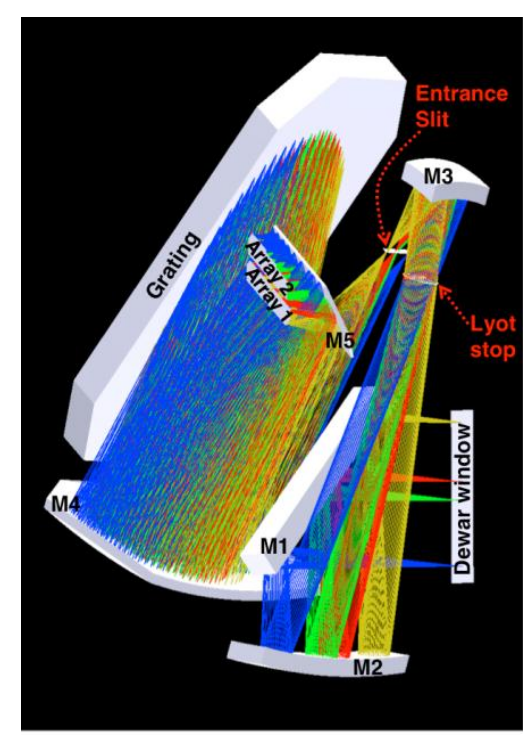

Figure 1: ZEUS-2 optics. Green and blue beams are spatial limits of Array 1, while yellow and red beams are spatial limits for Array 2/3.

\subsection{Instrument Design}

To achieve these goals, ZEUS-2 takes the optical design of ZEUS (see Figure 1) and marries it to several technological advances that push its capabilities significantly further. Namely, ZEUS-2 utilizes a pulse-tube refrigerator and adiabatic demagnetization refrigerator (ADR), and therefore requires no liquid cryogens, significantly reducing its operating costs

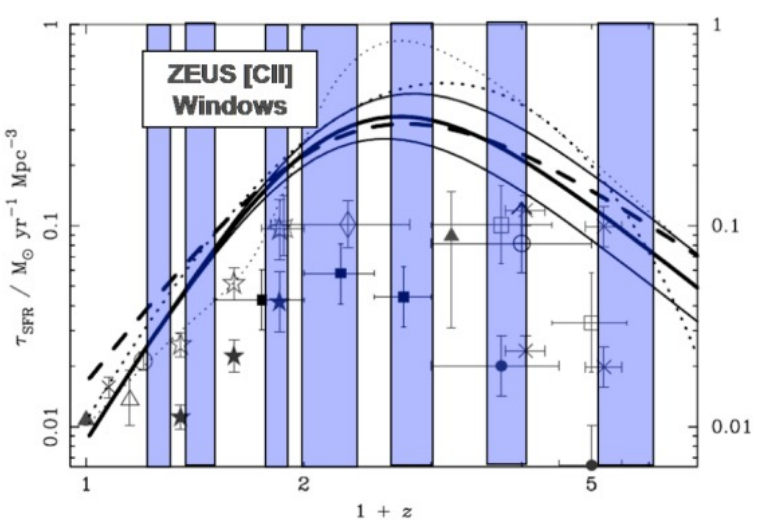

Figure 2: The co-moving star formation history of the Universe (S2). The original Madau plot based on optical/UV HDF observations are the filled marks. ${ }^{11}$ Open symbols correct this data for dust extinction. ${ }^{12}$ The 2 curves with error envelopes are models based on the SCUBA data. The transparent bars mark redshift ranges accessible in the $158 \mu \mathrm{m}$ [CII] line to ZEUS-2. and allowing the instrument to be used at sites with better atmospheric properties (see Parshley et al 2012, SPIE 8452-63, for a detailed description of the optical, mechanical, and thermal systems ${ }^{10}$ ). ZEUS-2 also features three state-of-the-art TES sensed bolometer arrays with tuned back-shorts allowing for $\sim 90 \%$ quantum efficiencies across 7 telluric windows from 200 to 850 microns (see Figure 2). The arrays allow for observations between 5 and 10 spatial positions on the sky and the simultaneous detection of five important submillimeter lines from extended sources or two lines from $z=1-2$ and 2.70 - 3 galaxies (see Figure 3). These key improvements make ZEUS-2 more sensitive than its predecessor by factors of 1.35 and 1.6 at 350 and 450 microns respectively (ZEUS-2's primary bands), increase the accessible redshifts by more than a factor of 2 , and produce a 10x improvement in mapping speed. Compared to other submillimeter spectrometers, ZEUS2 is the most sensitive submillimeter spectrometer. In its primary bands ZEUS- 2 is 3 to 5 times more sensitive than the best heterodyne receivers and 10 times more sensitive than the Herschel spectrometers. 


\section{DETECTOR}

\subsection{Array Design}

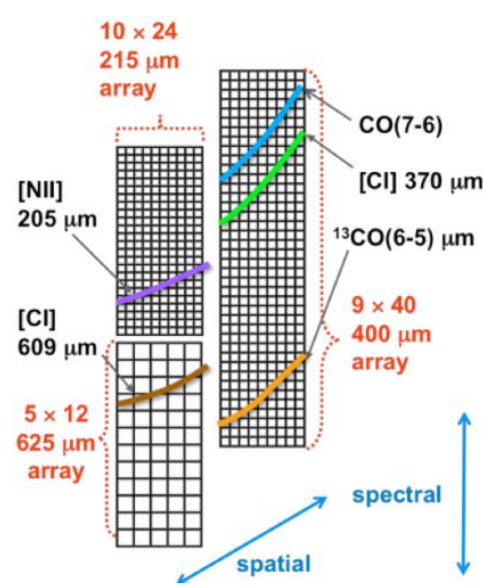

Figure 3: Schematic of ZEUS-2 arrays showing simultaneous detection of 5 important spectral probes.
The ZEUS-2 arrays are transition-edge-sensed bolometers from Kent Irwin's groups at NIST Boulder. The arrays are manufactured on two silicon wafers with arrays 2 and 3 sharing one wafer. The usable field-of-view (FoV) is $26 \mathrm{~mm}$ (spatial) x $57 \mathrm{~mm}$ (spectral) due to considerations of image quality. This usable FoV is split roughly equally between Array 1 and Array 2/3. The pixel sizes are 1.26, 1.06, and $2.06 \mathrm{~mm}$ square for Array 1, 2, and 3 respectively. For Array 1 and 2 there is a $0.14 \mathrm{~mm}$ structural/optical gap between each pixel $(0.2 \mathrm{~mm}$ for Array 3 ). Given that the final $\mathrm{f} / \#=2.75$, this corresponds to $1.15 \lambda / \mathrm{D}$ pixels at $400 \mu \mathrm{m}$ for Array 1. The array 2 pixels, however, are slightly oversized $(1.8 \lambda / \mathrm{D})$ to compensate for point source flux that would be lost with diffraction limited pixels on the Atacama Pathfinder Experiment (APEX) at $215 \mu \mathrm{m}$ due to surface roughness $(18 \mu \mathrm{m} \mathrm{rms})$, and the challenges of pointing with a 4.4" diffraction limited beam at $215 \mu \mathrm{m}$. The nominal format (spatial x spectral) of Array 1, 2, and 3 are $9 \times 40,10 \times 24$, and $5 \times 12$ pixels respectively. The actual array designs have the corner pixels cut-out (as can be seen in Fig. 8) so that the total pixel count is 555. These cut-outs provide space for handling the arrays as well as improved structural stability. Little science is lost by eliminating these pixels because poor image quality in these areas would have limited performance. Also, the spatial field is angled with respect to the spectral field and the cut-outs follow this angle.

To achieve a high detective quantum efficiency (DQE) all of the arrays feature a tuned backshort and palladium-gold mesh absorber. A high DQE is desirable because it improves the background limited noise equivalent power (NEP), and makes it easier to achieve background limited performance. The use of the mesh absorber is important as it allows for a high absorptive efficiency while adding very little to the heat capacity of the pixel. This is important if one wants to reach a low NEP while still maintaining a reasonable detector time-constant $(<10$ $\mathrm{msec})$. Modeling predicts that all arrays will operate with high quantum efficiency.

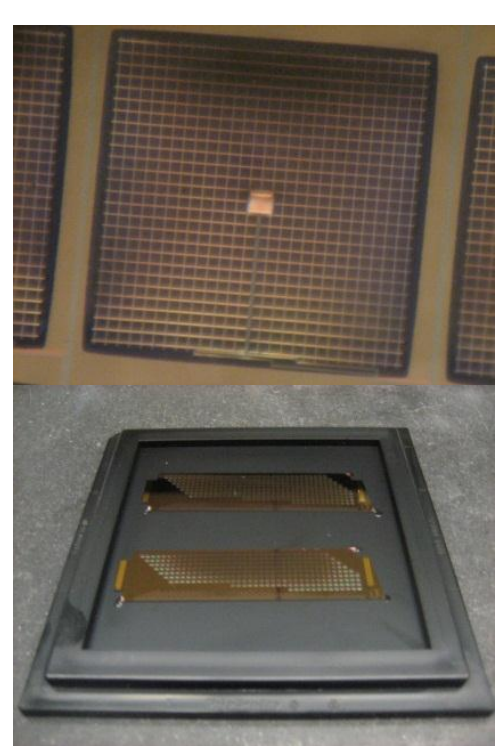

Figure 4: (top) Detailed picture of a ZEUS-2 pixel from the $400 \mu \mathrm{m}$ array showing the PdAu mesh absorber with $45 \mu \mathrm{m}$ pitch and TES. (bottom) Two ZEUS-2 science-grade $400 \mu \mathrm{m}$ arrays.

\section{$2.2400 \mu \mathrm{m}$ Array Design (Array 1)}

Array 1 is tuned to $400 \mu \mathrm{m}(\lambda / 4)$ resonance and will operate with better than $90 \%$ DQE in both the 350 and $450 \mu \mathrm{m}$ bands. Modeling of the absorber at NIST Boulder has provided straight forward mesh designs for Array 1 as seen in Figure 4. As the 350 and $450 \mu \mathrm{m}$ bands are ZEUS-2's primary bands, we have focused on implementing the $400 \mu \mathrm{m}$ array first. We have successfully tested in the lab and at the $\mathrm{CSO}$ an engineering grade array that had a pixel yield of $\sim 50$ percent (see Section 5). Science grade arrays with expected pixel yields $>90 \%$ have been received at Cornell and we are currently integrating them into the instrument

\subsection{5/645 $\mu \mathrm{m}$ Array Design (Array 2/3)}

Array $2 / 3$ is tuned to a $645 \mu \mathrm{m}(\lambda / 4)$ resonance. We expect that array 2 will see

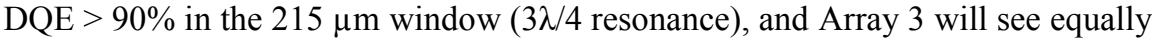
good DQE at the $645 \mu \mathrm{m}$ windows $(\lambda / 4)$, but at $850 \mu \mathrm{m}$ a DQE of $\sim 78 \%$ is expected. While modeling of the absorber at NIST Boulder has provided straight forward mesh designs for the longer wavelength arrays The $215 \mu \mathrm{m}$ required a more complex design. The final design features a mesh with $2 \mu \mathrm{m}$ lines on a 42 $\mu \mathrm{m}$ pitch made of $12 \Omega$ /square PdAu film giving an effective sheet impedance of $264 \Omega /$ square - close to the ideal impedance of free space, $377 \Omega /$ square. By adding a capacitively coupled square ring to each hole in the mesh, the sheet impedance better matches free space as well as removes parasitic reactance resulting in devices with less than 3.2\% reflection losses (Figure 5). Production of the first engineering grade versions of Array $2 / 3$ will begin soon. A full science grade Array $2 / 3$ will be installed early this fall. 

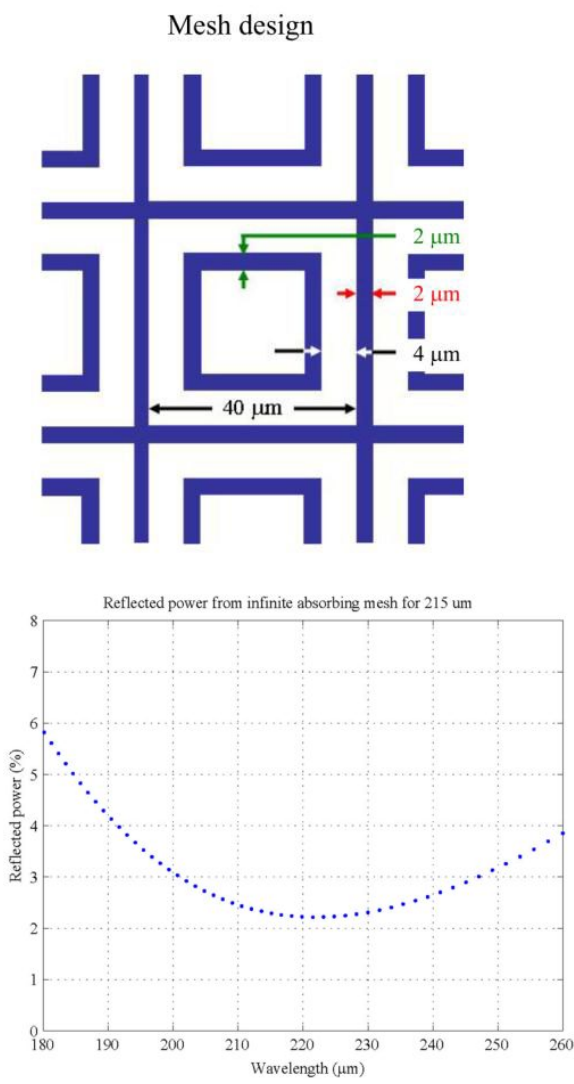

Figure 5: (top) PdAu mesh absorber design for Array 2 (bottom) Expected reflected power of Array 2

\subsection{Detector Package Design}

Developing a detector package that meets ZEUS-2's space limitations but at the same time fulfills the electronics requirements proved to be quite challenging. An initial design called for all 20 MUX chips, 20 interface chips (for interfacing the TES with the MUX chips and providing the shunt resisters for TES voltage biasing), and all three arrays on one 6" silicon wafer. A silicon wafer was chosen so that superconducting niobium traces could be patterned on the wafer to provide a superconducting interconnect between the arrays and the interface/MUX chips. Unfortunately there is not enough real-estate on a 6" wafer to fit all the necessary components and still leave room for traces and interfacing with cabling.

A novel solution presented itself however; separate the arrays and mount Array 1 and Array 2/3 on separate halves of the detector, with Array 1 and Array $2 / 3$ being respectively back and front illuminated. This creates a front and back focal plan that are then sandwiched together as in show in Figure 7. This design has the added benefit of enabling ZEUS-2 to be easily operated with only one array installed or with two of the same type. With two of Array 1installed, ZEUS-2 could observe 20 spatial positions on the sky in the 350 and $450 \mu \mathrm{m}$ bands. The arrays can also be easily swapped in and out for troubleshooting and upgrading of the bolometers. Even with the detector sandwich design, space was still tight; as such the detector package is designed to fully utilize the available space envelope, resulting in a very irregular shaped package as can be seen in Figure 6.

\subsubsection{Engineering Package}

Because the $400 \mu \mathrm{m}$ engineering-grade array featured relatively low pixel-yield, the detector package was populated with only a limited set of readout electronics. Namely, three MUX and three interface chips were used to read out a total of 84 pixels (see Figure 6). Electrical connections

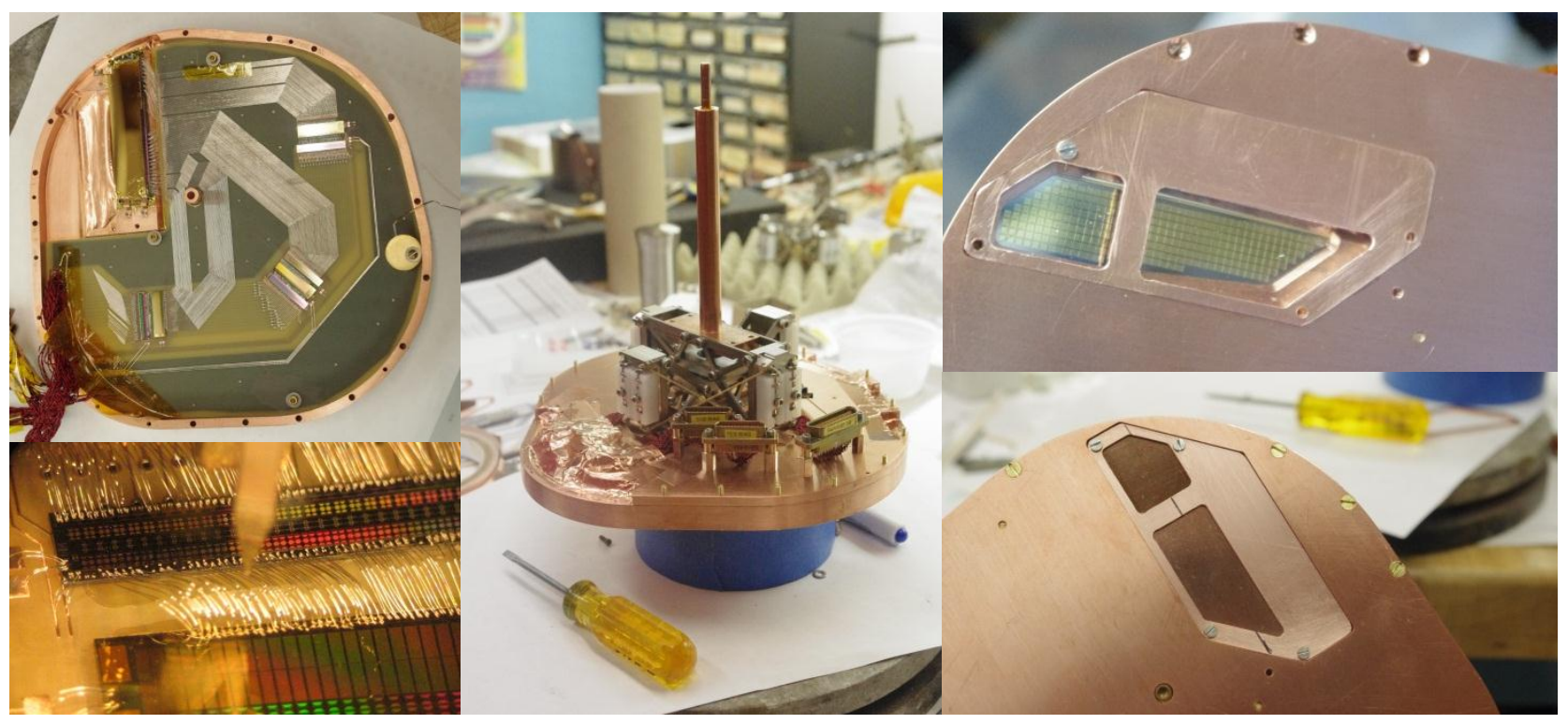

Figure 6: Starting in the upper left and moving clockwise: The open engineering-grade detector package; The fully assembled detector package before being enclosed in magnetic shielding; The detector and backshort through package entrance window; Entrance window covered with order-selecting bandpass filters; Al wirebonds between PCB, MUX chip, and interface chip 
to the MUX chips were provided by a standard multi-layer PCB. The TES to interface chip connection was also provided by the $\mathrm{PCB}$, but the traces were limited to the top surface and were lead-tinned so that the connection would be superconducting. The TES to PCB and MUX to PCB connections were made with Al wirebonds. Connections were made to the detector package and cable harness with twisted-pair wires soldered directly to the PCB and running to MDM connectors that in turn connect to the cable harness.

\subsubsection{Science Package}

The science-grade detector package, as show in Figure 7, will utilize the same copper box as the engineering package; however the internal electronics will be different. Each half of the package will house a PCB board for connection to ten MUX and interface chips, and a silicon fan-out board that provides a superconducting connection between the array and the interface chips. The array, with gold-coated back short, is mounted to the copper box with spring clips. A gold coated silicon frame between the array and box ensures that the array does not experience excessive thermal stress.
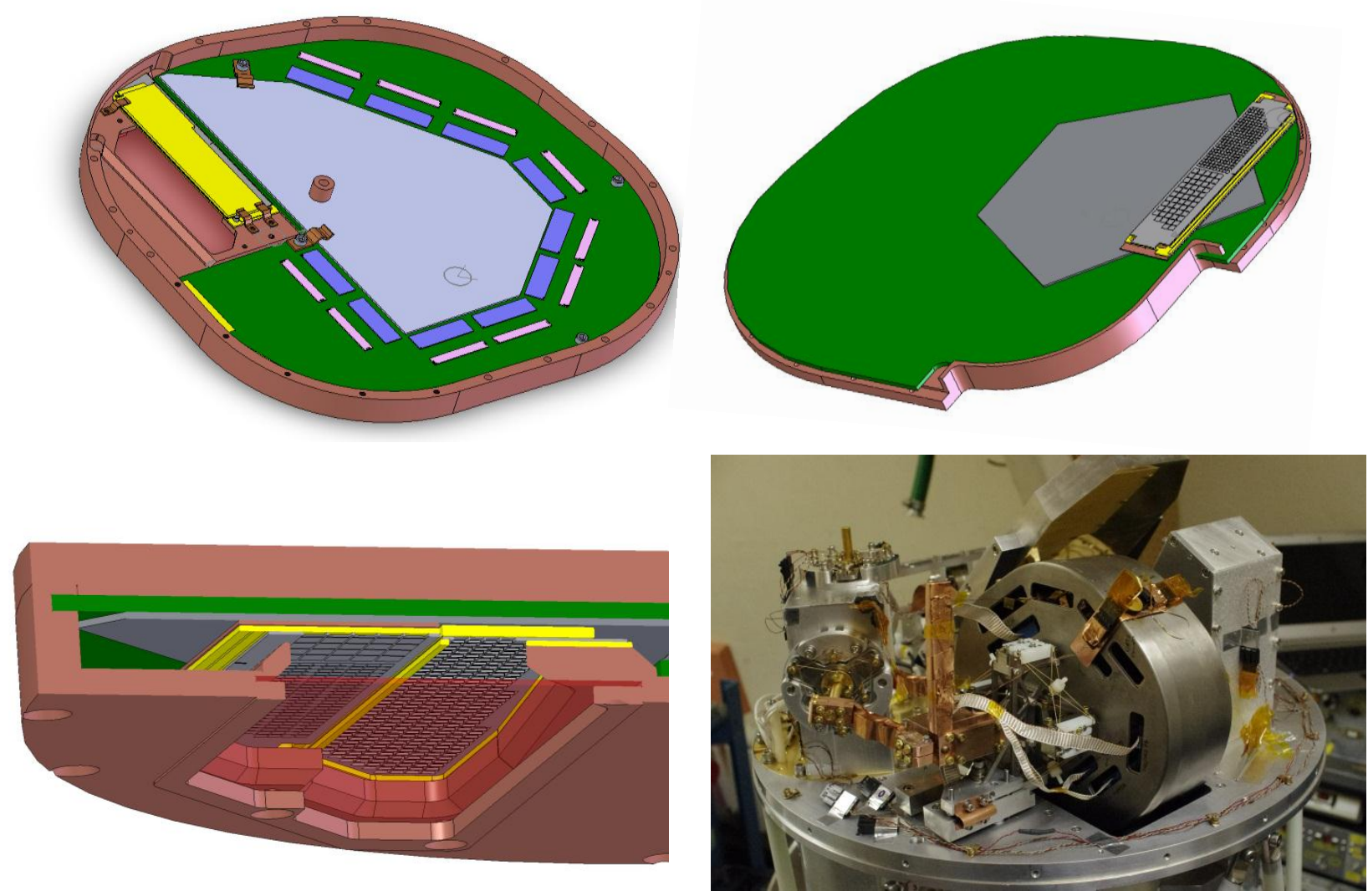

Figure 7: (top left) Front-half of the detector package for Array 1. (top right) Back-half of detector package for Array 2/3. (bottom right) Fully assembed detector package installed in ZEUS-2. (bottom left) A cut-away view of the assembled detector package. In all images: brown is the copper box, light-blue is the silicon fan-out board, dark blue are the interface chips, light purple are the MUX chips, and yellow are the gold coated backshorts and silicon carriers.

\section{MAGNETIC SHIELDING}

Because of SQUID sensitivity to magnetic fields, adequate magnetic shielding is a priority. ZEUS-2 features a highpermeability shield (Amumetal - Amuneal Mfg. Co.) just inside the vacuum shell to shield against fields sourced externally. Inside the dewar the ADR is designed with several shields that significantly attenuate the field when the ADR 
magnet is in operation. However, additional shielding is needed for the SQUIDs to remain functional. All SQUIDs, including the entire detector package, are housed in a A4K (Amuneal Mfg. Co.) shell surrounded by a superconducting niobium shell. This combination creates a zero field cavity around the SQUIDs once the niobium reaches it transition temperature of $7 \mathrm{~K}$, and as long as the magnetic field remains below 2000 Gauss (see Figure 8). Finite-Element-Analysis of the shielding predicted excellent performance. Operation and tests of the ZEUS-2 SQUID system confirmed these predictions.

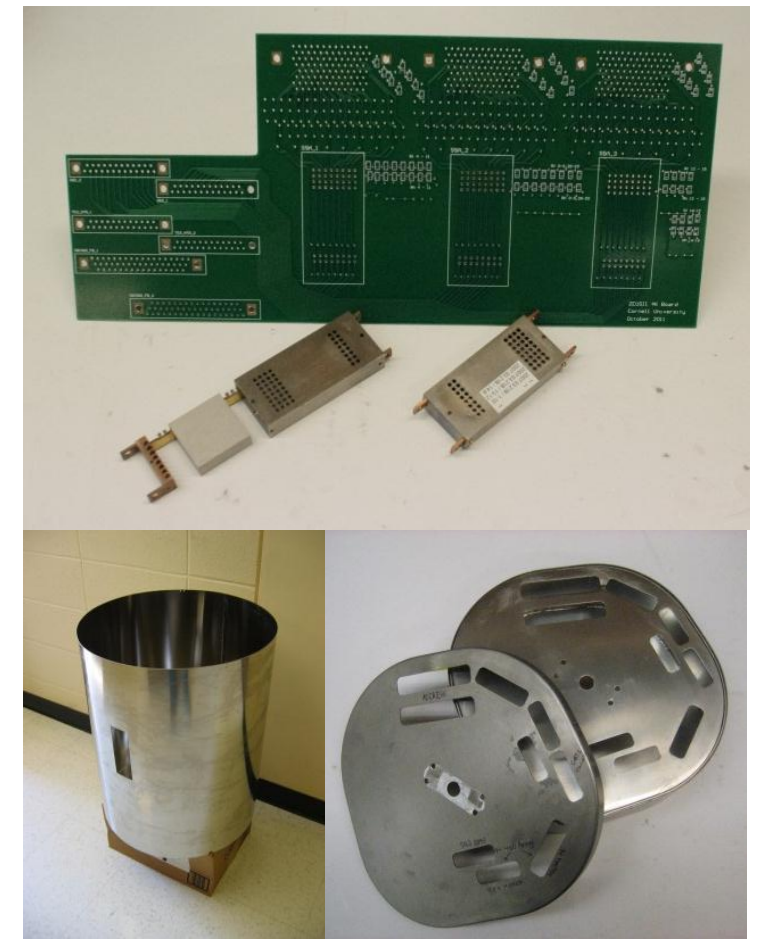

Figure 8: (top) 3-Kelvin PCB for SQUID Series Arrays and MCE-connector to detector fan-out board, and example Series Array modules. (bottrom right) The detector package magnetic shielding. (bottom left) The room temperature Amumetal magnetic shield.

\section{DETECTOR READOUT ELECTRONICS}

\subsection{Milli-Kelvin Electronics}

ZEUS-2 utilizes the three stage time domain SQUID multiplexed readout system developed at NIST Boulder. ${ }^{13}$ Each TES bolometer is coupled to one of the inputs on a 32-row SQUID multiplexor (20 MUX chips will be used in total). The multiplexors are housed in the detector package and their $1^{\text {st }}$ stage SQUIDS (SQ1) are coupled to the $3^{\text {rd }}$ stage SQUID series arrays (SQ3) by a $2^{\text {nd }}$ stage SQUID (SQ2).

\subsection{3-Kelvin Electronics}

The detector package is connected to 3-Kelvin electronics via copper-clad NbTi twisted pair Tekdata cables. The SQ2 to SQ3 interconnect uses two twisted pairs for each column to ensure that the inductance of that connection does not get too high and limit the multiplexing rate. The 3K-PCB houses the SQUID Series Arrays which amplify the detector signals by a factor of 100 before passing them over twisted-pair copper wires in four Tekdata cables to 100-pin MDM connectors mounted on the dewar shell.

\subsection{Multi-Channel Electronics (MCE) and Instrument Control Software}

Because of the SQUID sinusoidal response to a linearly changing input signal, an active feedback scheme must be used to ensure the SQUIDs are operating in their linear regime. ZEUS-2 uses the Multi-Channel Electronics (MCE) developed for SCUBA-2 by Mark Halpern's group at the University of British Columbia (UBC) to provide the active feedback. ${ }^{14}$ The MCE also provides the SQUID bias to power each stage of SQUIDS, the passive feedback for the SQ2 and SQ3, row addressing, pixel heater biases, and the TES biases. The MCE reads the TES response by using a PI control loop to servo the SQ1 feedback, canceling any change in the output of the TES and keeping the SQ3 output constant. It is the SQ1 feedback that is then the TES response and indicates the power incidence on the bolometer. The MCE is mounted directly the ZEUS-2 dewar (see Figure 9) and mates to the four 100pin MDM connectors on the dewar shell.

The MCE is controlled via a fiber connection by a computer running Linux. UBC provides an extensive suite of low level tools for running the MCE. We have incorporated these tools into a GUI program for easy operation at the telescope. The program also communicates with the telescope systems for obtaining information like the atmospheric transmission and to control the chopping and nodding. Since we observe in chopping mode, we perform a synchronous detection of the time series data using a reference signal from the secondary mirror that is sampled using the "Data_Valid" fiber connector of the MCE clock-card and custom clock-card firmware. When observing science sources we readout the detector at $400 \mathrm{~Hz}$ and take 16000 samples (40 seconds) for each plus or minus beam on the sky. A complete integration includes 32 beams, for a total of $\sim 21$ minutes. 


\section{PERFORMANCE AND FIRST LIGHT}

\subsection{In Lab Performance}

Several cool downs were performed to characterize the performance of the engineering-grade $400 \mu \mathrm{m}$ array. IV curves of the bolometers were taken with the array un-illuminated to determine the saturation power, thermal conductance, and transition temperature of the array. The transition temperature is $\sim 160 \mathrm{mK}$ and thermal conductance $\sim 60 \mathrm{pW} / \mathrm{K}$. At the operating temperature of $\sim 100 \mathrm{mK}$ the saturation power was $>$ than $2 \mathrm{pW}$, greater than twice that required to ensure that the bolometers do not saturate due to sky emission. In fact, the array is fully functional, with reasonable overhead, up to a bath temperature of $120 \mathrm{mK}$. This fact is important as operating the system at a high temperature can result in a significantly longer hold time for the ADR.
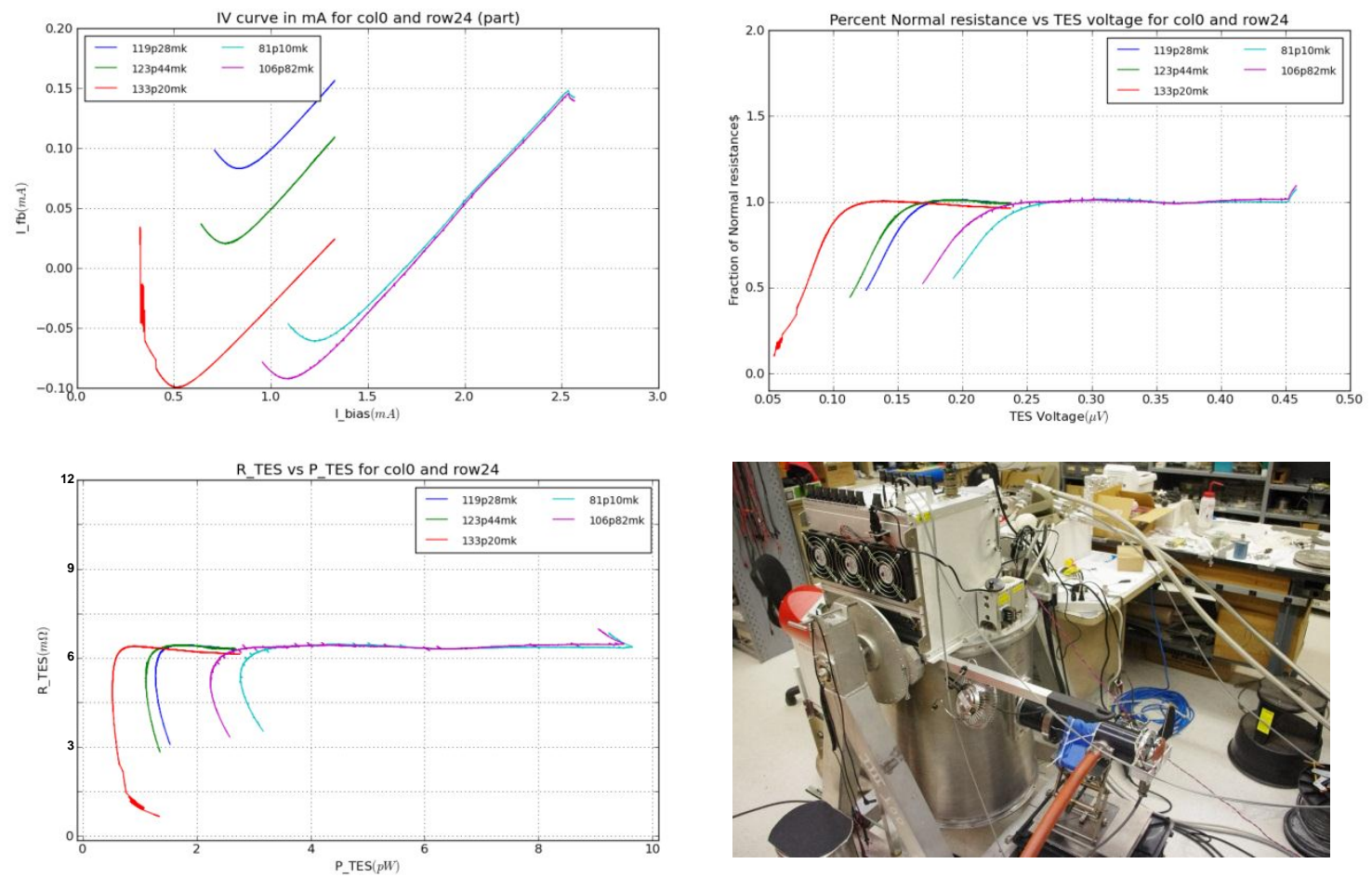

Figure 9: (top left) A dark IV curve for a representative $400 \mu \mathrm{m}$ pixel from the engineering-grade array showing the normal branch (linear region) and transition regions (non-linear part) for several bath temperatures. (top right) The percent normal-resistance versus the TES voltage. (bottom left) TES resistance versus the saturation power of the bolometer. (bottrom right) ZEUS-2 in the lab observing the CO gas cell.

Optical tests of the array were also successful. Measurements were made of a chopped liquid-nitrogen cold-load as well as a chopped 80-Celsius heater. The optical response to each was good. The noise, however, was higher than expecteda factor of 3-4 above the background limit. This higher noise is not an intrinsic part of the array, but is being injected into the detector though our house-keeping system. We are currently implementing solutions (i.e. changing the house keeping cabling) and fully expect to be background limited with the science grade array. Lastly a lab spectrum (Figure 10) was obtained by observing the chopped heater through a $\mathrm{CO}$ gas cell. 


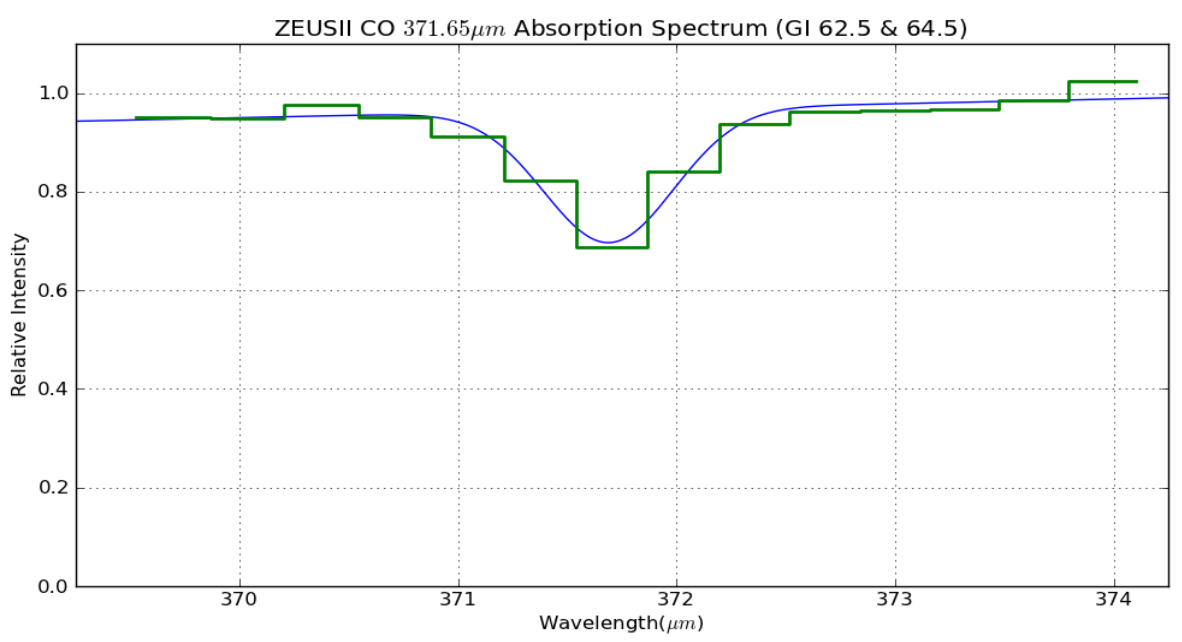

Figure 10: ZEUS-2 CO absorption spectrum

\subsection{First Light Observing Run}

Using the engineering-grade array, ZEUS-2 went on its inaugural observing run to the CSO in April 2012 (see Figure 11). The weather was idea for submillimeter weather $\left(\tau_{225 \mathrm{GHz}} \sim 0.05\right.$ or less). The instrument performed well on the telescope and in addition to the galactic sources we observed to characterize the instrument performance, we were able to observe $\sim 15$ different hig- redshift sources. We are currently reducing the characterization and science data to fully understand the instrument performance.

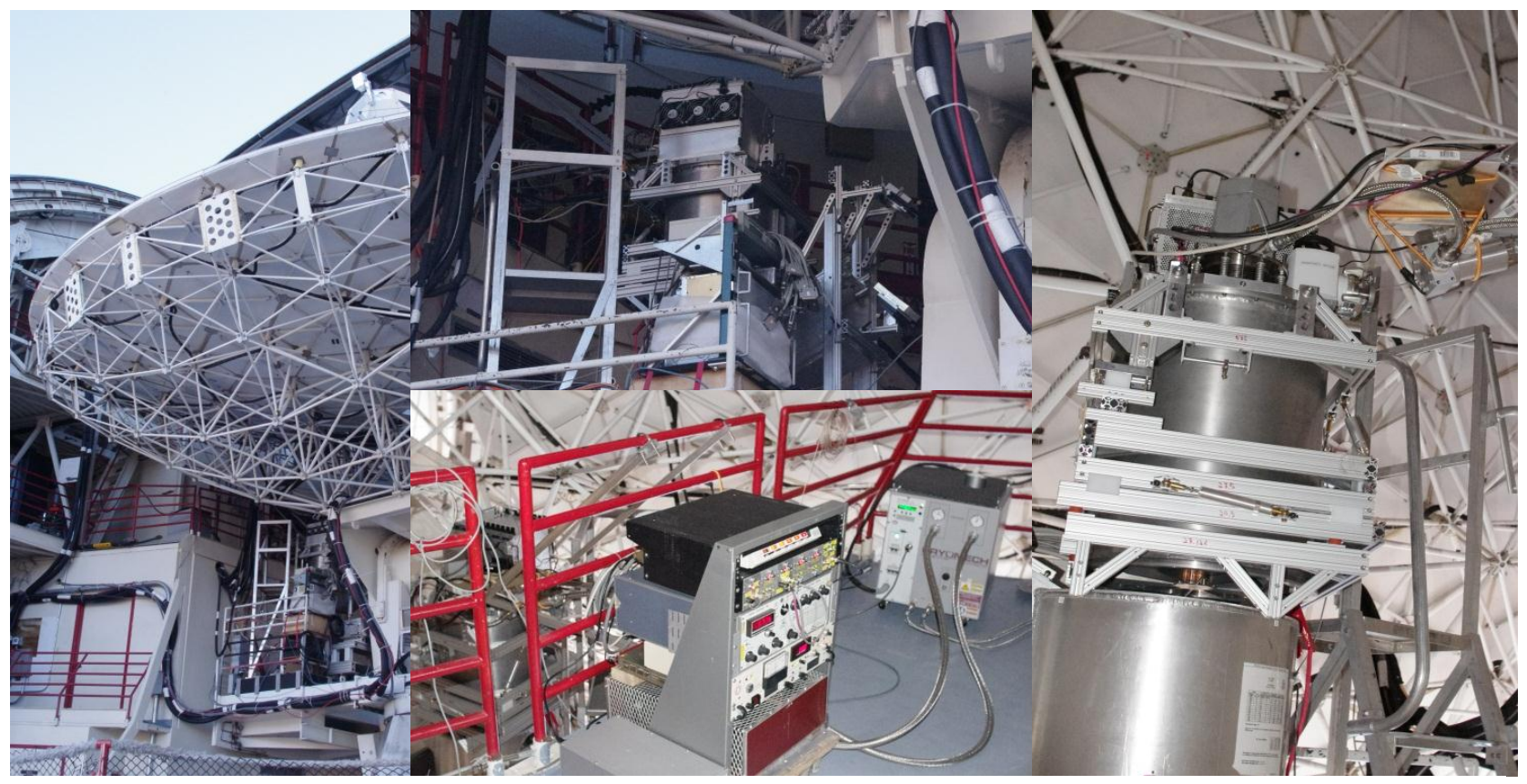

Figure 11: (left) ZEUS-2 on the nasmyth-2 platform at the CSO. (middle top) ZEUS-2 on the nasmith-2 platform clearing showing the MCE with three cooling fans, and fore-optics. (right) ZEUS-2 and mount at the CSO. (middle bottom) ZEUS-2 electronics rack and pulse-tube compressor at the CSO. ZEUS-2 is beyond the red railing on the left. 


\subsection{Sensitivity}

Here we describe the expected sensitivity of ZEUS-2. Based on models, lab tests, and first light performance we expect ZEUS-2 to be fully background limited once we have eliminated the excess noise do to the housekeeping system. The noise equivalent power, NEP of a background limited spectrometer with warm optical elements at temperature T, emissivity $\varepsilon$, and cold optical elements of transmission $\tau$ (emissivity 0 ) and DQE $\eta$, is given by, ${ }^{15}$

$$
N E P=h v \cdot\left\{\frac{2 A \Omega}{t_{\text {int }} \lambda^{2}} \cdot \Delta v \varepsilon \eta \tau \tilde{n} \cdot(1+\varepsilon \eta \tau \tilde{n})\right\}^{1 / 2} \text { Watts } \mathrm{Hz}^{-1 / 2} .
$$

Where $t_{\text {int }}$ is the integration time, $h$ is Planck's constant, $v$ is the frequency, $\Delta v=v / R$ is the detection bandwidth, $A \Omega / \lambda^{2}$ is the number of photon modes, $\tilde{\mathrm{n}}=1(\exp (\mathrm{hv} / \mathrm{kT})-1)$ is the mode occupation number. The factor of 2 accounts for detecting both polarizations of light. The noise equivalent flux, NEF, is then NEF $=2 \cdot \mathrm{NEP} /\left(\mathrm{A} \cdot \eta \cdot \tau \cdot \mathrm{t}_{\mathrm{w}}\right)$, where the factor of 2 is due to chopping loss, and $t_{w}$ is the warm transmission of the system. If at the front of the dewar the warm transmission is just $t_{w}=\eta_{\text {window }}$, the transmission of the polyethylene window $(92 \%)$. When considering an astronomical source, $t_{w}$ also includes the sky and telescope efficiencies, $\eta_{\text {sky }}(\lambda)$ and $\eta_{\text {tel }}(\lambda)$, as well as for point sources, the coupling to a pixel, $\eta_{\text {pix }}(\lambda)$.

Using equation 1 above, the expected point source sensitive for ZEUS-2 on CSO and APEX are listed for the band centers in Table 2 and plotted at all wavelengths in Figure 12. The current ZEUS sensitivities and the preflight sensitivity estimate for the Herschel instruments are also plotted. Because ZEUS-2 will be fully background limited and will have $1.28 \times$ better DQE, it is expected to be $\sim 1.35$ and 1.6 times more sensitive than ZEUS at 350 and $450 \mu \mathrm{m}$ bands.

Table 2: ZEUS-2/CSO and ZEUS-2/APEX Band Center System Parameters and Sensitivity

\begin{tabular}{|c|c|c|c|c|c|c|c|c|c|}
\hline $\begin{array}{l}\text { Band } \\
\text { Center } \\
(\mu \mathrm{m})\end{array}$ & $\begin{array}{l}\text { Slit } \\
\left({ }^{6}\right)\end{array}$ & $\begin{array}{c}\mathbf{R} \\
(\lambda / \Delta \lambda)\end{array}$ & $\eta_{\text {sky }}$ & $\eta_{\text {tel }}$ & $\begin{array}{c}\text { Power at } \\
\text { Detector } \\
\text { (Watts) }\end{array}$ & $\begin{array}{c}\text { NEP at } \\
\text { Detector } \\
\left(\mathrm{W} \mathrm{Hz}^{-1 / 2}\right)\end{array}$ & $\eta_{\text {pix }}$ & $\begin{array}{l}\mathrm{NEF}^{\mathrm{a}} \\
\left(\mathrm{W} \mathbf{m}^{-2}\right. \\
\left.\mathrm{Hz}^{-1.2}\right)\end{array}$ & $\begin{array}{c}\text { MDLF }^{b} \\
(5 \sigma, 4 h r s, \\
\left.\text { W m }{ }^{-2}\right)\end{array}$ \\
\hline APEX: & \multicolumn{9}{|c|}{$0.2 \mathrm{~mm}$ precipital water vapor (PWV) for $200-300 \mu \mathrm{m}$ bands, $0.5 \mathrm{~mm}$ at longer wavelengths } \\
\hline 202 & 5.6 & 1550 & 0.33 & 0.19 & $3.8 \mathrm{E}-12$ & $1.2 \mathrm{E}-16$ & 0.90 & $1.3 \mathrm{E}-16$ & $3.8 \mathrm{E}-18$ \\
\hline 233 & 6.2 & 1600 & 0.33 & 0.25 & $2.3 \mathrm{E}-12$ & $8.3 \mathrm{E}-17$ & 0.86 & $7.5 \mathrm{E}-17$ & $2.2 \mathrm{E}-18$ \\
\hline 295 & 6.9 & 1160 & 0.36 & 0.34 & $2.0 \mathrm{E}-12$ & $7.3 \mathrm{E}-17$ & 0.78 & $5.4 \mathrm{E}-17$ & $1.6 \mathrm{E}-18$ \\
\hline 354 & 7.5 & 980 & 0.53 & 0.40 & $1.2 \mathrm{E}-12$ & $5.3 \mathrm{E}-17$ & 0.71 & $2.3 \mathrm{E}-17$ & $6.8 \mathrm{E}-19$ \\
\hline 451 & 9.4 & 900 & 0.58 & 0.46 & $5.9 \mathrm{E}-13$ & $3.3 \mathrm{E}-17$ & 0.57 & $1.5 \mathrm{E}-17$ & $4.4 \mathrm{E}-19$ \\
\hline 626 & 15 & 580 & 0.60 & 0.50 & $8.3 \mathrm{E}-13$ & $3.5 \mathrm{E}-17$ & 0.76 & $9.4 \mathrm{E}-18$ & $2.8 \mathrm{E}-19$ \\
\hline 830 & 18 & 460 & 0.87 & 0.55 & $1.8 \mathrm{E}-13$ & $1.2 \mathrm{E}-17$ & 0.59 & $2.5 \mathrm{E}-18$ & $7.5 \mathrm{E}-20$ \\
\hline CSO: & \multicolumn{9}{|c|}{ Assumes $0.72 \mathrm{~mm}$ PWV } \\
\hline 354 & 8.7 & 980 & 0.38 & 0.41 & $1.5 \mathrm{E}-12$ & $5.9 \mathrm{E}-17$ & 0.71 & $4.1 \mathrm{E}-17$ & $1.2 \mathrm{E}-18$ \\
\hline 451 & 11 & 900 & 0.43 & 0.52 & $7.1 \mathrm{E}-13$ & $3.6 \mathrm{E}-17$ & 0.57 & $2.3 \mathrm{E}-17$ & $6.9 \mathrm{E}-19$ \\
\hline 626 & 17 & 580 & 0.46 & 0.64 & $1.1 \mathrm{E}-13$ & $3.8 \mathrm{E}-17$ & 0.76 & $1.3 \mathrm{E}-17$ & $3.9 \mathrm{E}-19$ \\
\hline 830 & 21 & 460 & 0.83 & 0.70 & $2.1 \mathrm{E}-13$ & $1.3 \mathrm{E}-17$ & 0.59 & $3.0 \mathrm{E}-18$ & $8.9 \mathrm{E}-10$ \\
\hline
\end{tabular}

${ }^{a}$ The NEF refers to the point source detection, i.e. it includes $\eta_{\text {pix }}$, the coupling to a pixel. ${ }^{b}$ MDLF is minimum detectable line flux $(5 \sigma)$ from a point source in 4 hours integration time. 
The predicted ZEUS-2 sensitivities significantly exceed the sensitivities of all current systems, including Herschel. Except at $200 \mu \mathrm{m}$, ZEUS-2 is about a factor of 10 more sensitive than the Herschel spectrometers. At $200 \mu \mathrm{m}$ ZEUS-2 is about equally sensitive to point sources as PACS, but has $\sim 2.5$ better spatial resolution. Currently, ZEUS has a equivalent heterodyne receiver temperature of $\mathrm{T}_{\text {rec }} \sim 20 \mathrm{~K}$ (single side-band), which is 4.6 and 2.3 times more sensitive than the best reported heterodyne values of $205 \mathrm{~K}$ (double side-band) and $250 \mathrm{~K}$ (single side-band) at 350 and $450 \mu \mathrm{m}$

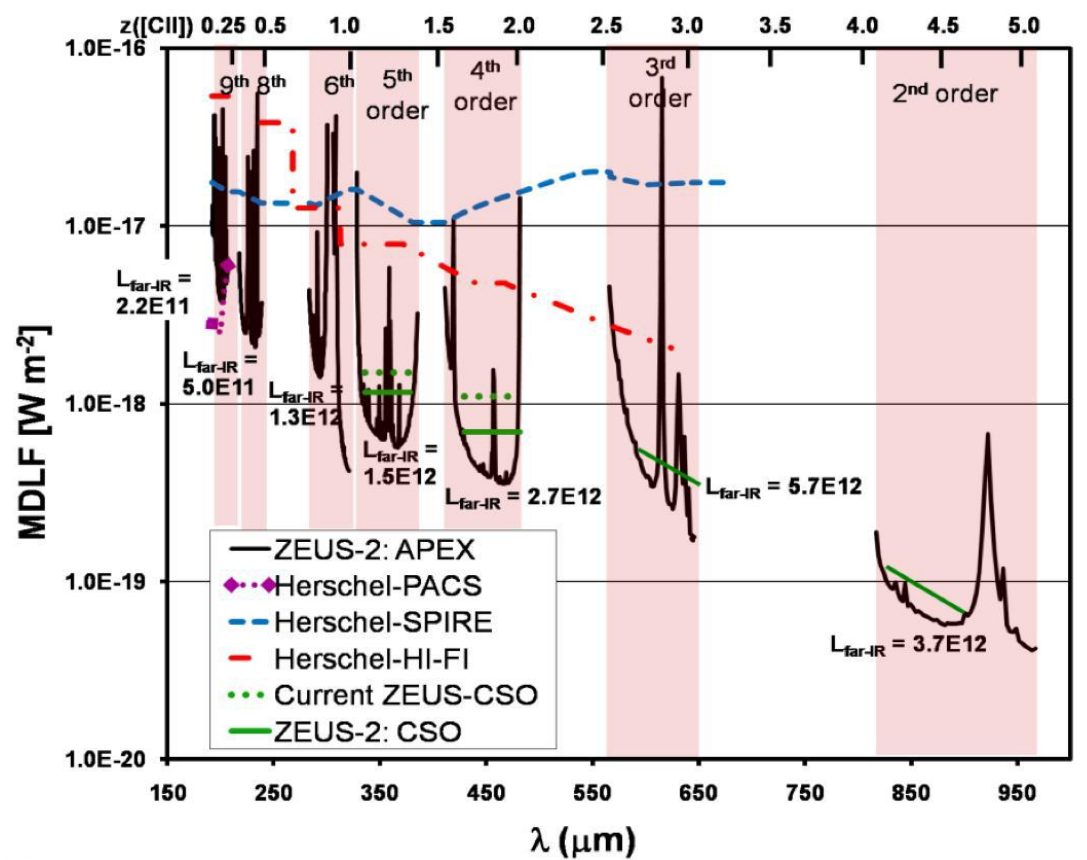

Figure 12: Minimum detectable line flux $\left(\mathrm{W} / \mathrm{m}^{2}\right)(5 \sigma, 4$ hours) for ZEUS-2 on APEX and on CSO. We use telluric transmission models with $0.5 \mathrm{~mm}$ and $0.72 \mathrm{~mm}$ PWV for APEX and CSO (at band center) respectively - appropriate for good submillimeter nights at their sites (APEX windows $<300 \mu \mathrm{m}$ use $0.2 \mathrm{~mm}$ ). Also plotted is the sensitivity of ZEUS, the published preflight sensitivities of the 3 spectrometers on the Herschel telescope ${ }^{16,17,18}$ with HIFI and PACS scaled to the resolving power of ZEUS-2: $\mathrm{R}=1000$, or $\Delta v=300 \mathrm{~km} \mathrm{~s}^{-1}$, appropriate for typical extragalactic line widths. ZEUS-2 is $\sim 10$ times more sensitive in the primary bands. The echelle orders, and the corresponding [CII] redshift intervals are listed, plus the minimum FIR luminosity galaxy detectable for each band assuming $\mathrm{R}=0.1 \%$ as we measure for the $\mathrm{z} \sim 1-2$ galaxies. We use $\mathrm{H}_{0}=71 \mathrm{~km} \mathrm{~s}^{-1} \mathrm{Mpc}^{-1}, \Omega_{0}=1, \Omega_{\Lambda}=0.7$.

respectively when the effects of the background are included (a $60 \%$ emissive, $270 \mathrm{~K}$ blackbody). ${ }^{19,20}$ Given its better sensitivity, we expect ZEUS-2 to be better than 3 and 5 times more sensitive than the best ground based heterodyne systems.

\section{SUMMARY}

The goal of ZEUS-2 is to study the star formation history of the universe from just a few billion years after the big bang to the present epoch. Building on the work of ZEUS, we will (1) investigate star formation from $\mathrm{z} \sim 0.25$ to 5 by measuring the redshifted fine-structure lines of distant galaxies, (2) measure redshifts from optically obscured galaxies by detecting the bright $158 \mu \mathrm{m}$ [CII] line, and (3) investigate the properties of nearby starburst and ultra-luminous galaxies through their submillimeter fine structure and $\mathrm{CO}$ rotational lines.

ZEUS-2 is an ideal instrument for this work as the moderate resolution ( $\mathrm{R} \sim 1000)$ of the long slit echelle grating spectrometer is optimal for detection of faint, broad lines from distant galaxies. Using three state-of-the-art TES bolometer arrays that are tuned for optimal efficiencies at 215, 400, and 625 microns, ZEUS-2 achieves sensitivities that are unparalleled from ground our space. When complete, ZEUS-2 will be able to obtain spectrum from up to nine spatial positions on the sky and have access to seven different bands between 200 and 850 microns allowing ZEUS-2 to excel at both mapping of nearby systems, and redshift searches of distant sources.

At present we successfully tested an engineering-grade $400 \mu \mathrm{m}$ array in the lab at the telescope. We are currently integrating our $400 \mu \mathrm{m}$ science-grade array in the instrument and will have a fully working Array 1 by the middle of Summer 2012. In early fall 2012 we will install our first engineering-grade Array $2 / 3$ into the instrument in preparation for our first observing run to APEX in October 2012. 
Acknowledgements: ZEUS development and observations were supported by NSF grants AST-0096881 and AST-0352855. ZEUS-2 development is supported by NSF grants AST-0705256 and AST-0722220 and NASA grant NNX10AM09H.

\section{REFERENCES}

[1] Hailey-Dunsheath, S., "Probing star formation at low and high redshift with ZEUS, a new submillimeter grating spectrometer", Ph.D. Thesis, Cornell University (2009) http://astro.cornell.edu/ spifiweb/zeus/publications/SHD_Thesis.pdf

[2] Hailey-Dunsheath, S., Nikola, T., Stacey, G.J., Oberst, T.E., Parshley, S.C., Bradford, C.M., Ade, P.A.R., \& Tucker, C.E., "Detection of the 13CO J=6-->5 transition in the Starburst Galaxy NGC 253", ApJ, Vol. 689, L109-L112, (2008).

[3] Ferkinhoff et al. First Detection of the [O III] $88 \mu \mathrm{m}$ Line at High Redshifts: Characterizing the Starburst and Narrow-line Regions in Extreme Luminosity Systems. The ApJ Letters (2010) vol. 714 pp. L147

[4] Ferkinhoff et al. First Detection of the [N II] $122 \mu \mathrm{m}$ Line at High Redshfit: Emonstrating the Utility of the Line for Studying Galaxies in the Early Universe. The ApJ Letters (2011) vol. 740 pp. L29

[5] Hailey-Dunsheath et al. Detection of the $158 \mu \mathrm{m}$ [C II] Transition at z $=1.3$ : Evidence for a Galaxy-wide Starburst. The ApJ Letters (2010) vol. 714 pp. L162

[6] Stacey et al. "A 158 micron [CII] line survey of galaxies at $\mathrm{z} \sim 1$ to 2 : an indicator of star formation in the early universe.” ApJ (2010) , vol. 724, pp. 957

[7] Nikola, T., Stacey, G.J., Hailey-Dunsheath, S., Ferkinhoff, C., Parshley, S., Oberst, T., Staguhn, J. and Benford, D. "Mid-J CO Emission from ULIRGs", near submission to ApJ, (2012).

[8] Nikola et al. Mid-J CO emission from NGC 891: Microturbulent Molecular Shocks in Normal Star Forming Galaxies, The Astrophysical Journal (2011) vol. 742, pp. 88

[9] Ferkinhoff et al., "ZEUS-2: a second generation submillimeter grating spectrometer for exploring distant galaxies", Millimeter, Submillimeter, and Far-Infraed Detectos and Instrumentation for Astronomy V. Proceedings of the SPIE, vol. 7741, pp. 77410Y (2010)

[10] Parshely et al.," The optical, mechanical, and thermal design and performance of ZEUS-2", SPIE 8452-63 (2012)

[11] Madau, P., Ferguson, H.C., Dickinson, M.E., Giavalisco, M., Steidel, C.C., Fruchter, A., "High-redshift galaxies in the Hubble Deep Field: colour selection and star formation history to z 4 “, Monthly Notices of the Royal Astronomical Society, Vol. 283, 1388 (1996)

[12] Pettini, M., Kellogg, M., Steidel, C.C., Dickerson, M., Adelberger, K.L., Giavalisco, M., "Infrared Observations of Nebular Emission Lines from Galaxies at Z = 3", ApJ, Vol. 508, 539 (1998)

[13] De Korte, P. A. J., Beyer, J., Deiker, S., Hilton, G., Irwin, K., MacIntosh, M., Nam, S. W., Reintsema, C., Vale, L. R., Huber, M. "Time-division superconducting quantum interference device multiplexor for transition-edge sensors." Rev. of Sci. Inst. Vol. 74, 8 (2003)

[14] Battistelli, E.S., Amiri, M., Burger, B., Halpern, M., Knoteck, S., Ellis, M., Gao, X., Kelly, D., MacIntosh, M., Irwin, K., Reintsema, C. "Functional Description of Read-out Electronics for Time-Domain Multiplexed Bolometers for Millimeter and Sub-millimeter Astronomy." J Low Temp Phys. (2008) 151: 908-914

[15] Bradford, C.M., Stacey, G.J., Swain, M.R., Nikola, T., Bolatto, A.D., Jackson, J.M., Savage, M.L., Davidson, J.A., \& Ade, P.A.R., "SPIFI: a direct-detection imaging spectrometer for submillimeter wavelengths", Applied Optics, 41, 2561 (2002)

[16] de Graauw, T., Whyborn, N., Helmich, F., Dieleman, P., Roelfsema, P., Caux, E., Phillips, T., Stutzki, J., Beintema, D., Benz, A., Biver, N., Boogert, A., Boulanger, F., Cherednichenko, S., Coeur-Joly, O., Comito, C., Dartois, E., de Jonge, A., de Lange, G., Delorme, I., DiGiorgio, A., Dubbeldam, L., Edwards, K., Fich, M., Güsten, R., Herpin, F., Honingh, N., Huisman, R., Jacobs, H., Jellema, W., Kawamura, J., Kester, D., Klapwijk, T., Klein, T., Kooi, J., Krieg, J.-M., Kramer, C., Kruizenga, B., Laauwen, W., Larsson, B., Leinz, C., Liseau, R., Lord, S., Luinge, W., Marston, A., Merkel, H., Moreno, R., Morris, P., Murphy, A., Naber, A., Planesas, P., Martin-Pintado, J., Olberg, M., Orleanski, P., Ossenkopf, V., Pearson, J., Perault, M., Phillip, S., Rataj, M., Ravera, L., Saraceno, P., Schieder, R., Schmuelling, F., Szczerba, R., Shipman, R., Teyssier, D., Vastel, C., Visser, H., Wildeman, K., Wafelbakker, K., Ward, J., Higgins, R., Aarts, H., Tielens, X., Zaal, P., "The Herschel-Heterodyne Instrument for the Far-Infrared (HIFI): instrument and pre-launch testing", in Space Telescopes and Instrumentation 2008: Optical, Infrared, and 
Millimeter, Eds. Oschmann, J.M., Jr., de Graauw, M.W.M., MacEwen, H.A., Proceedings of the SPIE, Vol. 7010, pp. 701004-701004-14 (2008)

[17] Griffin, M., Swinyard, B., Vigroux, L., Abergel, A., Ade, P., André, P., Baluteau, J.-P., Bock, J., Franceschini, A., Gear, W., Glenn, J., Huang, M., Griffin, D., King, K., Lellouch, E., Naylor, D., Oliver, S., Olofsson, G., PerezFournon, I., Page, M., Rowan-Robinson, M., Saraceno, P., Sawyer, E., Wright, G., Zavagno, A., Abreu, A., Bendo, G., Dowell, A., Dowell, D., Ferlet, M., Fulton, T., Hargrave, P., Laurent, G., Leeks, S., Lim, T., Lu, N., Nguyen, H., Pearce, A., Polehampton, E., Rizzo, D., Schulz, B., Sidher, S., Smith, D., Spencer, L., Valtchanov, I., Woodcraft, A., Xu, K., Zhang, L., "Herschel-SPIRE: design, ground test results, and predicted performance", in Space Telescopes and Instrumentation 2008: Optical, Infrared, and Millimeter, Eds. Oschmann, J.M., Jr., de Graauw, M.W.M., MacEwen, H.A., Proceedings of the SPIE, Vol. 7010, pp. 701006-701006-12 (2008)

[18] Poglitsch, A., Waelkens, C., Bauer, O.H., Cepa, J., Feuchtgruber, H., Henning, T., van Hoof, C., Kerschbaum, F., Krause, O., Renotte, E., Rodriguez, L., Saraceno, P., Vandenbussche, B., "The Photodetector Array Camera and Spectrometer (PACS) for the Herschel Space Observatory", in Space Telescopes and Instrumentation 2008: Optical, Infrared, and Millimeter, Eds. Oschmann, J.M., Jr., de Graauw, M.W.M., MacEwen, H.A., Proceedings of the SPIE, Vol. 7010, pp. 701005-701005-12 (2008)

[19] Kooi, J.W., Kawamura, J., Chen, J., Chattopadhyay, G., Pardo, J.R., Zmuidzinas, J., Phillips, T.G., Bumble, B., Stern, J.A., \& LeDuc, H.G., "A Low Noise NbTiN-Based 850 GHz SIS Receiver for the Caltech Submillimeter Observatory", International Journal of Infrared and Millimeter Waves, 21, 1357 (2000)

[20] Güsten, R., Baryshev, A., Bell, A., Belloche, A., Graf, U., Hafok, H., Heyminck, S., Hochgürtel, S.,Honingh, C.E., Jacobs, K., Kasemann, C., Klein, B., Klein, T., Korn, A., Krämer, I., Leinz, C., Lundgren, A., Menten, K.M., Meyer, K., Muders, D., Pacek, F., Rabanus, D., Schäfer, F., Schilke, P., Schneider, G., Stutzki, J., Wieching, G., Wunsch, A., Wyrowski, F., "Submillimeter heterodyne arrays for APEX", in Millimeter and Submillimeter Detectors and Instrumentation for Astronomy IV. Eds. Duncan, W.D., Holland, W.S., Withington, S., Zmuidzinas, J., Proceedings of the SPIE, Vol. 7020, pp. 702010-702010-12 (2008) 